\title{
COMENTARIO A VARRÓN LING. V 131-133. ¿UNA CLASIFICACIÓN POCO RIGUROSA DE LAS PRENDAS DE VESTIR, O UN REFLEJO DE LA MENTALIDAD INDUMENTARIA ROMANA?
}

The author proposes a new interpretation of Varr. Ling. V 131-133. The anaysis of spatial notions (extra/intus and supra/subter) and the order in which the passage is disposed allows us to see in it a reflection of the Roman clothing system.

\section{O. Introducción}

El pasaje de Varrón De lingua latina V 131-133 está dedicado tanto a la etimología como a la clasificación de las prendas romanas. Aunque uno y otro aspecto son interesantes, lo es especialmente el segundo, pues Varrón articula el pasaje en torno a dos tipos de prenda que denomina mediante dos formaciones en - tu-, indutui y amictui, que se corresponden, asimismo, con dos verbos de vestir, induo y amicio:

(1) Varr. Ling. V 131-133: Prius deinde indutui, tum amictui quae sunt tangam. Capitium ab eo quod capit pectus, id est, ut antiqui dicebant, comprehendit. Indutui alterum quod subtus, a quo subucula; alterum quod supra, a quo supparus nisi id quod item Osce. Alterius generis item duo, unum quod foris ac palam, palla; alterum quod intus, a quo indusium, id quod Plautus dicit:

Indusiatam patagiatam caltulam ac crocotulam.

Multa post luxuria attulit, quorum uocabula apparet esse Graeca, ut asbestinom.

Amictui dictum quod ambiectum est, id esta circumiectum, a quo etiam quo uestitas se inuoluunt, circumiectui appellant, et quod amictui habet purpuram circum, uocant circumtextum. Antiquissimi amictui ricinium; id quod eo utebantur duplici, ab eo quod dimidiam partem retrorsum iaciebant, ab reiciendo ricinium dictum.

Pallia hinc, quod facta duo simplicia paria, parilia primo dicta, $r$ exclusum propter leuitatem. Parapechia, clamydes, sic multa, Graeca. Laena, quod de lana multa, duarum etiam togarum instar; ut antiquissimum mulierum ricinium, sic hoc duplex uirorum.

Esta división de los vestidos en aquellos que se 'introducen por el cuerpo' (indutui) y aquellos que lo 'rodean' (amictui) presenta algunas dificultades de 
traducción a una lengua moderna ' y Collart, en su conocido comentario al libro $\mathrm{V}$ de Varrón, considera que es una clasificación poco rigurosa ${ }^{2}$, a lo que se suman, asimismo, las incoherencias habidas dentro de los lexicógrafos latinos en la caracterización de las prendas. Sin embargo, como en otro lugar ${ }^{3}$ hemos observado, se trata de una distribución efectiva en el plano de la lengua que responde al tipo de indumentaria usada en la Antigüedad, dentro de culturas donde era corriente el uso de túnicas adheridas al cuerpo y de mantos o togas envolventes. Así, en lo que a la cultura romana respecta, es posible una clasificación, en términos generales, según el criterio de que la prenda se ponga introduciéndola por el cuerpo, como la túnica, o bien colocándola en derredor del mismo, como la toga. Este hecho guarda relación con la existencia en latín de dos verbos específicos para expresar la colocación de uno u otro tipo de prenda. Así, para las prendas que se introducen, como la túnica, se utiliza induo 'poner introduciendo y adaptando', y para las prendas que rodean el cuerpo, por su parte, se usa amicio, cuyo valor originario es 'poner la prenda por ambos hombros'. Ambas acciones indumentarias conforman la manera de vestir romana y pueden verse expresadas conjuntamente en algunos pasajes de la literatura latina (p. e. V. Max. II 7, 9 toga laciniis abscissis amictum, discincta tunica indutum).

Por otra parte, la clasificación tanto de verbos como de prendas según los criterios de 'intromisión' y 'en derredor' no debe confundirse con otra clasificación que posiblemente es más familiar para nosotros, precisamente la que divide las prendas en inferiores (subter) y superiores (supra), pues si bien es verdad que la prenda que rodea al cuerpo está por encima, esta noción es pertinente en especial para algunas de las prendas que se introducen en él, como es el caso de las túnicas que se ponen sobre la túnica inferior o subucula.

\footnotetext{
I Son variadas las traducciones que ha recibido el comienzo del pasaje, unas tratando de ser literales, y otras intentando interpretar el sentido último de la clasificación:

«Maintenant, je vais traiter d'abord la question des vêtements ajustés, puis celles des vêtements drapés» (J. C. Collart, ed., Varron, De lingua latina. Livre V, París 1954, p. 87); «Next I shall first touch upon those things which are for putting on, then those which are for wrapping about the person" (R. G. Kent, ed., Varro, On the Latin language I, Londres y Cambridge (Mass. 1958, p. 125; "Ora io traterio prima la questione dei vestiti che s'indossano, poi di quelli con cui si avvolga, sopra di quisti, la persona» (A. Taglia, ed., M. T. Varrone, Opere, Turin 1974, p. 139); "A continuación voy a tratar de los vestidos corrientes, y luego de la ropa de abrigo" (Marcos Casquero, ed., Varrón, De lingua Latina, Madrid 1990, p. 99).

${ }^{2}$ Collart, op. cit., p. 229, n. 1: «L'oposition de indutui avec amictui est, en principe, celle de vêtements qu'on met sur soi, en passant la tête ou le bras pour des ouvertures (indutui), avec les vêtements que l'on drape (amictui). Cette classification est loin d'être rigoureuse.»

${ }^{3}$ Cf. F. García Jurado, Los verbos de "vestir" en la lengua latina (Introducción al lenguaje indumentario), Amsterdam, Hakkert, 1995, pp. 36-54.
} 
Entendemos, pues, que el pasaje de Varrón puede volver a interpretarse a la luz de tales presupuestos, tanto en lo que respecta a la clasificación de las túnicas como a las incoherencias habidas entre los lexicógrafos latinos y los mismos testimonios de Varrón a la hora de caracterizarlas.

Nuestro trabajo se divide en dos partes: en 1 haremos un estado de la cuestión sobre la interpretación de las túnicas, y en 2 analizaremos el pasaje varroniano objeto de este trabajo. En 3, finalmente, estableceremos las conclusiones del análisis.

1. La clasificación de las túnicas romanas y sus problemas. Estado de la cuestión

Las túnicas romanas son diversas, pero tienen un aspecto común, pues debemos pasar e introducir los brazos y la cabeza por ellas para adaptarlas, en mayor o menor medida, a nuestro cuerpo. Ya hemos dicho que esta acción indumentaria se expresa en latín con el verbo induo, y permite establecer un tipo de prendas que pueden denominarse genéricamente como uestes induendae. Es, precisamente, dentro de este tipo de prendas donde vamos a encontrar diversos problemas de interpretación en torno a su caracterización. Como es sabido, junto a la denominación más genérica y extendida de este tipo de prendas, tunica, la lengua latina cuenta con otros nombres que designan variedades de túnica más concretas, en especial el indusium, la subucula (junto a la que debemos considerar también la inducula) y el supparus. Esta diversidad está relacionada con el hecho de que a partir de cierto momento los romanos comenzaron a llevar dos túnicas y, lo que es más importante para nosotros, las denominaran de diferente manera, tal y como nos lo refiere Varrón:

(2) Varr. in Non. 870 L.: SVBVCVLA. Varro de uita Populi Romani lib. I: posteaquam binas tunicas habere coeperunt, instituerunt uocare subuculam et indusium.

El pasaje ha recibido más de una lectura, pero, según la más aceptada, debemos entender que una vez que los romanos comenzaran a llevar dos túnicas, aquella que quedaba debajo se denominó subucula 'túnica inferior', mientras que la que quedaba por encima pasó a llamarse indusium. Sin embargo, los testimonios latinos que veremos seguidamente y, en especial, el pasaje del mismo Varrón ya citado en (1), no parecen ser congruentes con lo que el pasaje (2) nos dice, y ello ha dado lugar, a su vez, a opiniones discordantes por parte de los estudiosos modernos. Vamos a realizar una revisión detenida de cada prenda: 


\subsection{Indusium}

El indusium es un tipo de túnica que, según la interpretación más general del testimonio de Varrón arriba citado (2), queda por encima de la subucula y cuyo origen, por lo demás, es incierto ${ }^{4}$. Pasando ya al resto de los testimonios antiguos, Varrón nos ofrece otra definición del indusium, ahora en el pasaje ya citado de su De lingua latina:

(3) (=1) Varr. Ling. V 131: alterum quo intus, a quo indusium ut intusium, id quod Plautus dicit:

indusiatam ${ }^{5}$, patagiatam, caltulam aut crocotulam.

Como puede verse, Varrón hace hincapié, en este caso, en el carácter interior ${ }^{6}$ de la túnica, algo que en principio, no parece congruente con la idea de túnica superior que veíamos en la definición del pasaje (2). Junto a los dos testimonios de Varrón ya citados, el término indusium aparece comentado en Nonio, quien parafrasea la última definición varroniana y hasta repite el ejemplo:

(4) Non. p. 866 L.: INDVSIVM est uestimentum quo corpori intra plurimas uestes adhaeret, quasi intusium. Plautus in Epidico (231):

indusiatam, patagiatam, caltulam aut crocotulam.

Por su parte, los comentaristas modernos interpretan así la prenda, sobre la base de los testimonios antiguos:

4 Véase un buen estado de la cuestión en A. Ernout, "Indusium, indusiarius, indusiatus», $R P h$ 32, 1958, pp. 7-8 y, más recientemente, en A. Potthoff, Lateinische Kleidungsbezeighnungen in syncroner und diachroner Sicht, Innsbruck 1992, pp. 123-127. Lo que sí parece claro es que indusium no puede relacionarse con formas derivadas de induo como indutus o indumentus, pues, frente a la - u - larga de éstos, la de indusium es breve. Por otra parte, Ernout (Les éléments dialectaux du vocabulaire latin, París 1929, p. 184) señala que la $-s$ - intervocálica pone a indusium en relación con alguno de los dialectos itálicos donde ésta se haya mantenido más tiempo que en latín. La hipótesis que relaciona indusium con gr. Ěvঠovors [así A. Walde y J. B. Hofmann, Lateinisches etymologisches Wörterbuch, Heidelberg, $1982_{5}$ (en adelante W.-H.) $s$. $u$., pero con reservas], presenta el problema, en buena medida insalvable, de la aparición tardía del término griego.

5 Para la morfología de indusiatam con respecto a indusium cf. Ernout, art. cit., pp. 9 y $13-14$.

6 Otra interpretación posible de intus es la de una prenda que se usa dentro de la casa, sobre la subucula (cf. J. Marquardt, Das Privatleben der Römer, II, Leipzig, 1882, p. 468). La misma confrontación que hace Varrón con la palla, vestido que se lleva palam, podría ser un hecho a favor de esta interpretación (cf. Potthoff $o p$. cit., p. 124). No obstante, no debemos olvidar que se trata de un intento, entre los otros que veremos en este trabajo, por salvar la incongruencia de los testimonios varronianos habida entre (1) y (2). Por otra parte, el ejemplo plautino aducido en el pasaje (Plaut. Epid. 231) para la tunica indusiata no dice nada a favor de la etimología intus que Varrón sostiene, ni en el sentido de túnica interior, ni en el de túnica de casa. Sabido es que este pasaje Palutino del Epidicus pertenece a una crítica misógina acerca de la variedad de prendas que ostentan las mujeres, una de las cuales es la tunica indusiata. 
- Marquardt ${ }^{7}$ define el indusium como el vestido que llevan las mujeres sobre la subucula. Collart coincide asimismo en su carácter de túnica superior ${ }^{8}$ frente a la subucula ${ }^{9}$.

- Frente a la unanimidad de los comentarios anteriores, Wilson, en su monografia ya clásica sobre la indumentaria romana ${ }^{10}$, considera, por el contrario, al indusium como una 'under tunic'. Esta interpretación trata de salvar, sin aludir a ello explícitamente, la aparente contradicción dada entre los testimonios latinos para indusium citados, pues, por un lado, Varrón (2) da a entender que el indusium es la prenda que aparece sobre la subucula, en el caso de que ésta quede por debajo y, por otro lado, Varrón (3) (=1) y luego Nonio (4) consideran esta prenda como interior (intra plurimas uestes adhaeret). Wilson intenta resolver la aparente aporía mediante una nueva interpretación del pasaje (2), según la cual subucula e indusium son lo mismo en lo que respecta a su carácter de 'under tunic'. La traducción que nos da del pasaje es, por lo demás, significativa:

After they began to have two tunics, they started the custom of calling (the under one, evidently) subuculam or indusium ".

Como puede verse, para poder llevar a cabo esta interpretación, la estudiosa norteamericana se ha visto obligada a introducir un paréntesis aclaratorio, con el fin de especificar que ambos términos conciernen a la «túnica de debajo» $y$, por otra parte, ha tenido que traducir la conjunción copulativa et del texto latino por una conjunción disyuntiva, todo para tratar de equiparar el indusium a la subucula, y salvar así las incongruencias. En nuestra opinión, son demasiados «retoques» los que precisa esta interpretación. Ha sido comúnmente aceptada, por el contrario, la explicación más sencilla, aquella que precisamente opone y no equipara el indusium (tunica superior) a la subucula (tunica inferior), a pesar de que no se haya encontrado hasta ahora coherencia con los otros dos testimonios (3) y (4) ${ }^{12}$.

- Todos estos problemas dan lugar a la que podemos considerar la postura escéptica, muy bien recogida por Ernout en el pasaje siguiente:

7 J. Marquardt, op. cit., p. 468.

${ }^{8}$ Collart, op. cit., p. 229, n. 3: «les compilateurs son d'accord pour nous présenter l'indusium como une 'tunique de dessus'".

9 «Subucula désigne la 'tunique de dessous' et s'oppose à indusium qui a concurrencé inducula».

${ }^{10}$ L. M. Wilson, The Clothing of the ancient Romans, Baltimore 1938, pp. 71 y 165.

1 Wilson, op. cit., p. 71.

12 Otro intento por resolver el problema viene de la mano de Riposati (M. Terenti Varronis De Vita Populi Romani, Milán 1972, p. 161), y que comentaremos en el apartado dedicado a la subucula. 
Nous ne savons pas quelle sorte de vêtement désignait au juste l'indusium. Si l'on croit le fragment de Varron qui l'oppose à subucula, ce serait un vêtement de dessus; mais, dans le De lingua latina, ce même Varron explique indusium par intus en fait un vêtement intèrieur; explication que Nonius reproduit religieusement ${ }^{13}$.

Pero es conveniente analizar, a su vez, el par subucula-inducula y supparus para extraer una idea global del problema:

\subsection{Subucula $e$ inducula}

Varrón, además del pasaje en el que refiere el uso de dos túnicas a partir de un momento dado (2), vuelve a aludir a la subucula ${ }^{14}$ en tres nuevas ocasiones:

(5) a. (=1) Varr. Ling. V 131: indutui alterum quod subtus, a quo subucula.

b. Varr. Ling. IX 46: itaque in uestitu in supellectile delectari uarietate, non paribus subuculis uxoris.

c. Varr. in Non. p. 880 L.: quo (sc. palliolo) mulieres nunc (...) utuntur, postquam subuculis desierunt.

Las interpretaciones modernas han coincidido unánimente en lo que concierne a la posición inferior que ocupa esta prenda, salvo Riposati, quien, en un nuevo intento por hacer corresponder los diferentes testimonios varronianos, considera que la subucula es la prenda que está bajo la toga, mientras que el indusium es la túnica que se introduce, según su opinión, por debajo de la subucula $^{15}$. Esta interpretación pretende ser tan fiel al testimonio varroniano que invierte la lectura más general que venimos viendo. Entre las variadas objeciones que se le pueden hacer, debemos tener en cuenta, sobre todo, que es más fácil e inteligible que la determinación 'por debajo' de la subucula guarde relación con el indusium, pues es la prenda a la que se opone directamente, más bien que con la toga. En este sentido, es muy revelador el siguiente pasaje de Horacio alusivo, asimismo, a la subucula:

(6) Hor. Ep. I 1,95: si forte subucula pexae trita subest tunicae.

En él se especifica claramente la posición de las túnicas (subucula subest tunicae), aunque el término indusium se encuentre aquí sustituido por el más general de tunica. Por otra parte, la toga y los mantos, no se caracterizan por estar 'encima' de otras prendas, sino por estar 'en derredor' del cuerpo, es

13 A. Ernout, art. cit., p. 12.

14 Para los problemas etimológicos de subucula e inducula véase la nota 19.

15 «Sono quidi due sottovesti, di cui la subucula veniba indossata 'subito dopo la toga e l'indusium rimaneva aderente al corpo, quasi una camisia'» (Riposati, op. cit., p. 161; cf. también Riposati, «Nota varroniana sull'antico abbigliamento romano», RIL 103, 1969, pp. 755-761). 
decir, por otra noción espacial, como veremos más adelante, y este hecho, en apariencia irrelevante, tiene una importancia clave en la mentalidad indumentaria romana. El resto de interpretaciones, como hemos dicho, coinciden en el carácter de prenda inferior de la subucula con respecto al indusium:

- Marquardt ${ }^{16}$ considera que es una túnica interior, así como Collart ${ }^{17}$, quien define a la subucula como una 'tunique de dessous', a la que opone el indusium (cf. 1.1), 'tunique de dessus', término este último que, en su opinión, ha desplazado a inducula.

- Wilson cree igualmente que la subucula es una 'under tunic', aunque, como hemos visto (1.1), también considera 'under tunic' al indusium. Para diferenciarlas de alguna manera, hace la salvedad de que aquélla sería utilizada por los hombres, frente al indusium, la 'under tunic' que llevarian las mujeres, pero se muestra escéptica con su propia caracterización ${ }^{18}$. Por otra parte, ya hemos considerado los serios problemas que la interpretación y traducción de Wilson con respecto al pasaje varroniano traía consigo.

Otro problema no menos interesante viene dado por la relación entre subucula e inducula ${ }^{19}$. Si bien hay consenso en la caracterización de la subucula

16 Op. cit., pp. 468 y 535.

17 Op. cit., p. 229.

18 "Since the other name, subucula, as stated above, seems to have been appropriated by the man's tunic, indusium has been generally accepted as the name of the corresponding garment of the matron. The evidence in both cases is inferential rather than positive, and it is probable that there was no hard and fast rule for the application of either name, and that both were often used generically, indicating under tunics in general» (Wilson, op. cit., pp. 165-166).

${ }_{19}$ Inducula 'túnica, vestido' y subucula 'túnica de debajo' presentan una serie de interesantes problemas relacionados con su significado y etimología. Inducula, desarrollo sufijal a partir de induo, es un hapax plautino (Plaut. Epid. 223); se acepta normalmente (W. H., $s$. u. subucula) que, al igual que subucula, está formado por un sufijo instrumental *-tla, o, en términos de Serbat (Les dérivés nominaux latins à suffixe médiatif, París, 1975, pp. 145-146), mediativo, aunque Ernout (art. cit., p. 12), por su parte, cree que se trata de un sufijo diminutivo. En lo que respecta a subucula, el problema más arduo es el que concierne al origen de su formación. Por una parte, la explicación más sencilla, tal y como creen Ernout y Meillet [Dictionnare étymologique de la langue latine. Histoire des mots, París, 19794, (en adelante E. M.) s. u. exuo], es suponer que se haya formado sobre la base de inducula, con el problema que conlleva hacer derivar un término que aparece atestiguado en todas las épocas de la latinidad a partir de un hapax. Por esta razón, Schwyzer («Miszellen", RhM 76, 1927 , p. 444, n. 1) y W. H. (s. u. exuo y subucula) consideran inducula como una creación personal de Plauto, y, a su vez, proponen como forma base para subucula un desarrollo con sufijo instrumental y sin preverbio de la raíz *eu: *ucula (*ou-tla cf. lit. àutla 'dünne Leine' E. Fraenkel, Litauisches Etymologisches Wörterbuch, Heidelberg, 1962, s. u. aùtli-, av. ao@ra 'Schuhzeug'). Por otra parte, rechazan de plano la hipótesis de un supuesto verbo *sub-uo, no atestiguado en ninguna parte. Serbat (op. cit., pp. 145-146), después de hacer un estado de la cuestión, vuelve a la explicación de subucula a partir de inducula, apelando al posible cruce de esta palabra con indusium y a que inducula haya podido aparecer más veces de las que los textos recogen. Véase un último estado de la cuestión en Potthoff, op. cit., p. 185. 
como una «tunique de dessous», Serbat, en la última revisión del término realizada hasta la fecha, por lo que sabemos, no comprende cómo puede justificarse este nombre de prenda a partir del de inducula ${ }^{20}$. Sin embargo, en nuestra opinión, la oposición dada entre los preverbios sub- e ind-propios de un nombre y de otro, lejos de no ser clarificadora, es significativa, como veremos a lo largo de este trabajo. Así mientras sub-en sub-ucula implica la posición 'por debajo' 21 [recuérdese el testimonio horaciano (6)], ind- en inducula implica el acto de intromisión (induo) de la prenda junto al cuerpo, que no ha de estar necesariamente por debajo de otra. De esta forma, sub- e indno designan lo mismo en este caso, ni tampoco responden a la misma noción espacial.

\subsection{Supparus}

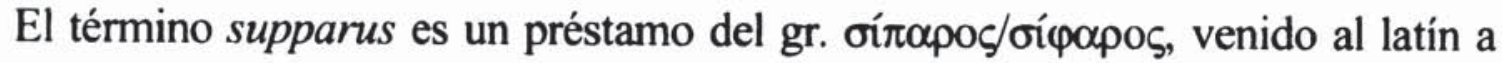
través del osco, tal y como el mismo Varrón reconoce ${ }^{22}$. El tipo de prenda que este término designa es especialmente objeto de interpretaciones contradictorias:

Varrón le aplica el carácter de túnica superior y trata de buscar su etimología en supra-:

(7) (=1) Varr. Ling. V 131: alterum quod supra, a quo supparus, nisi id quod item dicunt Osce.

${ }^{20}$ Cf. Serbat, op. cit., pp. 145-146: «Il ne serait donc pas imposible que inducula aix mieux existé qu'il ne semble d'après le textes. Mais comment justifier subucula à partir de lui? Inducula signifiant simplement 'ce que l'on revêt', 'le vêtement', l'opposition des préfixe sub- et ind-n'est pas éclairante. On comprendrait un *sub-inducula. Mais subucula ne signifie rien à partir de seul inducula.»

${ }_{21}$ García Hernández (Semántica estructural y lexemática del verbo, Barcelona-Reus 1980 , pp. 200-208) ha estudiado las tres realizaciones espaciales del preverbio sub-, causa de frecuentes confusiones:

- Noción direccional 'hacia arriba':

sub-/de- "desde arriba"

suspendo/dependet

succingo

- Noción posicional vertical 'debajo':

sub-/super- 'por encima'

subsum/supersum

subucula/superuestio

- Noción posicional horizontal 'por detrás':

sub-/prae- 'por adelante'

suppono/praepono

Para la distinción entre el carácter posicional y direccional de los preverbios cf. idem, pp. 223-224.

${ }^{22}$ Ernout, op. cit., pp. 234-235. Véase la última puesta al día de los problemas etimológicos de supparus en Potthoff, op. cit., pp. 186-190. 
Sin embargo, Nonio define el supparus como prenda inferior ${ }^{23}$ :

(8) Non. p. 866.: SVPPARVM est linteum femorale usque ad talos pendens dictus quod subtus appareat. Plautus in Epidico (232):

supparum aut subnimium (est), ricam, basilicum aut exoticum.

Como vemos por el texto, la identificación que hace Nonio del supparus como túnica inferior se basa en un pasaje del Epidicus plautino, a partir del hapax sub-parum (sobre un falso corte de supparum) que forma parte de un juego de palabras, con el fin de crear el neologismo sub-nimium. De esta forma, se establece, una oposición entre una supuesta túnica, 'insuficiente' (parum) y una túnica 'excesiva' (nimis). La interpretación del preverbio sub- en este caso puede ser diversa, y no necesariamente la de 'por debajo' que parece sugerir Nonio Marcelo. Así, Ernout ${ }^{24}$ cree que el nombre de la túnica denominada supparum ha recibido una interpretación popular a partir del falso corte dado entre sub- y parum, lo que la ha relacionado con el verbo supparo 'ajustar', sólo atestiguado en Tertuliano. Pero puede darse otra interpretación si nos atenemos a que Plauto utiliza la expresión adverbial de la insuficiencia y el exceso para la creación del hapax ${ }^{25}$. Sub-parum, así interpretado, da a entender una prenda insuficiente, en lo que al supuesto adverbio parum respecta, $\mathrm{y}$, en nuestra opinión ${ }^{26}$, el también supuesto preverbio $s u b$-, en el caso de interpretarse como una realización diminutiva ${ }^{27}$. En definitiva, el preverbio sub- en el juego plautino sub-parum no es, de manera alguna, un argumento a favor del carácter inferior de la prenda.

${ }^{23}$ Es probable que Nonio esté confundiendo subtus 'por debajo - de otra prenda (cf. Hor. Ep. I, 95) - ' con la parte inferior del cuerpo. Hay dos indicios bastante claros para pensar que esto es así: en primer lugar, la referencia a una parte del cuerpo como talos (usque ad talos pendens) y, en segundo lugar, el hecho de que la prenda pueda verse al sobresalir por debajo (quod subtus apparet). Esta noción espacial que considera la parte inferior o superior del cuerpo o el vestido es distinta, pues, a la que venimos viendo aquí para explicar la túnica inferior (la que está debajo de la túnica superior), y está menos representada en el lenguaje indumentario latino, aunque hay ejemplos significativos de ella:

Tac. Germ. 17, 3: feminae... partem... uestitus superioris in manicas non extendunt, nudae bracchia ac lacertos.

Curt. III 1, 38: dein summa quaque amicula exuunt (...) ad ultimum (...) ima corporum uelamenta proiciunt.

Non. p. 869 L.: LIMBVS, ut adnotatum inuenimus, muliebre uestimentum, quod purpuram in imo habet.

${ }^{24}$ Art. cit., pp. 7-14.

25 Precisamente, el asunto de la insuficiencia y el exceso ornamental femenino articula la crítica misógina contra el adorno femenino en la comedia, como puede verse en diversos recursos léxicos utlizados con tal fin (cf. F. García Jurado, "La critica al exceso oramental femenino en la comedia latina a partir de los recursos léxicos relativos a la Lex Oppian, Minerva 6, 1992, pp. 193-208).

${ }^{26}$ Cf. García Jurado, op. cit., en n. 3, p. 104.

27 Para esta realización véase García Hernández, op. cit., p. 205. 
Festo, por su parte, equipara el supparus a la subucula, y Paulo Diácono, en su epítome de Festo, explica ésta por medio de otra prenda en principio ajena a la cultura romana, pero del mismo carácter, como es la camisia ${ }^{28 .}$

(9) Paul. Fest. p. 406 L.: supparus uestimentum puellare lineum, quod et subucula, id est camisia dicitur. Afranius (122):

Puella non sum, supparo si induta sum?

Las interpretaciones modernas reproducen las mismas discrepancias:

- Marquardt considera que el supparus es una prenda superior que llevaban las mujeres ${ }^{29}$.

- Collart ${ }^{30}$, asimismo, cree que se trata de una 'túnique de dessus' muy amplia que desciende hasta los pies.

- Ernout ${ }^{31}$, por el contrario, considera que el supparus es un sinónimo de subucula.

- Finalmente, Wilson ${ }^{32}$ da la razón a Festo y considera, por tanto, que el supparus es un 'undergarment'. A su vez, hace notar que ha sido precisamente a partir de la autoridad de Varrón por la que supparus se traduce a veces por 'manto', 'chal', u otras prendas exteriores. Ejemplo significativo de ello es este tardío testimonio literario que encontramos en Lucano, acerca de la segunda boda de Catón y Porcia. En este pasaje, supparus ha recibido tradicionalmente la traducción de 'chal' 33 :

(10) Luc. II 360-364: non timidum nuptae leuiter tectura pudorem lutea demissos uelarunt flammea uoltus, balteus aut fluxos gemmis astrinxit amictus,

28 "Qua Paulus adiicit: id est camisia, sumpsit ex suorum temporum more et semone.» [Sextus Pompeius Festus, De uerborum quae supersunt cum Pauli epitome, emendata et annotata a C. Odofredo Mueller, Hildesheim 1975 (Lipsiae 1839), p. 311, n. 12].

29 Marquardt, op. cit., p. 468: «Es bezeichnet (sc. supparus) ein Oberkleid, welches Frau beim Ausgehen anlegten". Asimismo, Pottohff (op. cit., pp. 186 y 234) lo define como "Kleidungsstück der Frau». En verdad, son varios los testimonios que asocian el supparus al uso femenino (Plaut. Epid. 232, Afran. com. 123, Luc. II 364, Paul. Fest. p. 406 L.), lo que no debe de ser casual. No obstante, no olvidemos que la inducula, el indusium y la subucula también aparecen relacionados con las mujeres: así, tanto inducula como tunica indusiata pueden verse junto al supparus en Plaut. Epid. 223 y 231, respectivamente, pasaje misógino alusivo a los excesos ornamentales femeninos (cf. $\mathrm{nn} .6$ y 25), y la subucula también se asocia a las mujeres en Varr. Ling. IX 46 y Varr. in Non. p. 880 L. Véanse, asimismo, los problemas que planteaba la adscripción que hacía Wilson (op. cit., pp. 16566) del indusium y la subucula a sexos diferentes.

${ }^{30}$ Op. cit., p. 229.

31 Art. cit., p. 11.

${ }^{32}$ Op. cit., pp. 154-165.

33 Commenta Bernensia, ed. Usener, p. 72: «Subpara pro amiculis. 'Suppara' genus uestis quod alii stolam dicunt, alii toracem, uel amiculi genus. Alii 'uestes angustae sine manicis pube tenus quibus uirgines nupturae induuntur'.» 
colla monile decens umerisque haerentia primis suppara nudatos cingunt angusta lacertos.

\subsection{Cuadros}

En definitiva, parece que lo único evidente es la divergencia habida en las interpretaciones de los nombres de túnica comentados, tanto en los antiguos lexicógrafos como en los comentaristas modernos, tal y como los dos cuadros siguientes nos muestran, a manera de resumen:

CUADRo I. Lexicógrafos latinos

\begin{tabular}{|l|l|l|l|c|}
\hline & \multicolumn{2}{|c|}{ Varro } & \multicolumn{1}{c|}{ Non. } & Paul. Fest. \\
\hline indusium & intus $(1)(=3)$ & supra $(2)$ & intra $(4)$ & \\
\hline subucula & subtus $(1)(=5 \mathrm{a})$ & subtus $(2)$ & & \\
\hline supparus & supra $(1)(=7)$ & & subtus $(8)$ & = subucula $(9)$ \\
\hline
\end{tabular}

CUADRO II. Estudiosos modernos

\begin{tabular}{|l|l|l|l|l|l|}
\hline & \multicolumn{1}{|c|}{ Marquardt } & \multicolumn{1}{|c|}{ Collart } & \multicolumn{1}{|c|}{ Serbat } & \multicolumn{1}{c|}{ Ernout } & \multicolumn{1}{|c|}{ Wilson } \\
\hline indusium & $\begin{array}{l}\text { tunica } \\
\text { interior }\end{array}$ & $\begin{array}{l}\text { tunique de } \\
\text { dessus }\end{array}$ & & $?$ & $\begin{array}{l}\text { under } \\
\text { tunic }\end{array}$ \\
\hline subucula & $\begin{array}{l}\text { tunica } \\
\text { inferior }\end{array}$ & $\begin{array}{l}\text { tunique de } \\
\text { dessous }\end{array}$ & $\begin{array}{l}\text { sous } \\
\text { vêtement }\end{array}$ & $\begin{array}{l}\text { sin. de } \\
\text { supparus }\end{array}$ & $\begin{array}{l}\text { under } \\
\text { tunic }\end{array}$ \\
\hline supparus & Oberkleid & $\begin{array}{l}\text { tunique de } \\
\text { dessus }\end{array}$ & & $\begin{array}{l}\text { sin. de } \\
\text { subucula }\end{array}$ & $\begin{array}{l}\text { under } \\
\text { garment }\end{array}$ \\
\hline
\end{tabular}

Varias razones influyen en este estado de cosas. Vamos a especificar seguidamente las tres que nos parecen más importantes:

a) Los testimonios de Varrón (2) y (1) $(=3)$ son la causa principal de las divergencias en lo que a la interpretación del indusium concierne.

b) La divergencia del testimonio de Varrón (1) (=7) frente a Non. (8) y Paul. Fest. (9) es la causa principal de la interpretación ambigua del supparus. Esta divergencia interpretativa de Varrón con respecto a Nonio, Festo y Paulo Diácono puede estar motivada por la considerable distancia cronológica de un autor con respecto a los otros (Varrón, s. I a. C.; Nonio, s. IV d. C.; Festo, s. II - finales-, y Paulo Diácono, s. VIII).

c) La polisemia del preverbio sub- y su interferencia con el preverbio in(d) — 'intromisión' cuando sub - expresa la noción espacial 'por debajo'. Es importante tener en cuenta que no todas las uestes induendae se caracterizan por se inferiores, como es el caso de la subucula. 
Planteado, pues, este complejo estado de la cuestión, podemos pasar ya al análisis del pasaje varroniano que nos ocupa.

\section{El pasaje varroniano}

El pasaje del De lingua latina de Varrón (1) que revisa los distintos tipos de prenda está implicado directamente en los problemas que tratamos aquí. Dentro de lo impreciso que puede ser este tipo de testimonios, encontramos que el polígrafo latino divide, como ya hemos comentado al comienzo, los tipos de prenda en aquellas que se ponen 'introduciéndolas por el cuerpo' (indutui) y las que se ponen 'rodeándolo' (amictui). En lo que a este segundo tipo de prendas respecta, es significativo que Varrón haga hincapié precisamente en el hecho de que estas prendas rodeen y envuelvan al cuerpo (amiectum est, id est circumiectum, a quo uestitas se inuoluunt), sin necesidad de hacer uso de la noción 'por encima' (supra), que si bien es en la realidad extralingüística una noción coincidente con la de «rodear el cuerpo con una prenda", no es, sin embargo, la noción pertinente para estas prendas ${ }^{34}$. Esta circunstancia hace especialmente llamativo el hecho de que Varrón reserve supra para uno de los dos subtipos de prendas indutui, según estén situadas por debajo (subtus) o por encima (supra):

(11) (=1) Varr. Ling. V 131: (...) Capitium ab eo quod comprehendit pectus, id est ut antiqui dicebant, capit. Indutui alterum quod subtus, a quo subucula; alterum quod supra, a quo supparus, nisi id quod item dicunt Osce. Alterius generis item duo, unum quod foris ac palam, palla; alterum quod intus, a quo indusium ut intusium, id quod Plautus dicit:

indusiatam, patagiatam, caltulam ac crocotulam

En este pasaje, más que las discutibles etimológicas ad hoc (supra: supparum; palam: palla; intus: indusium), es la disposición del material léxico comentado lo que puede tener un interés relevante para reinterpretar un texto que se ha considerado tradicionalmente como poco riguroso, pero que quizá no sea tan incoherente como se cree. Tras hablar del capitium, prenda que, efectivamente, se adscribe a induo ${ }^{35}$, observamos, pues, que Varrón establece dos géneros de prendas indutui (primum genus y alterum genus): las de debajo

${ }^{34}$ Será en latín postclásico cuando la noción de super 'por encima' adquiera importancia, en detrimento de la noción 'en derredor' (circum-, amb-) (cf. García Jurado, op. cit., en n. 3, pp. 59-60).

${ }^{35}$ Así podemos verlo en el siguiente fragmento de Laberio:

Laber. com. 61: induis capitium, tunicae pittacium.

Las capuchas y cobertores para la cabeza, así como el calzado, se clasifican como prendas indutui, por su adaptación a una parte del cuerpo (cf. García Jurado, op. cit., en n. 3, p. 40). 
(subucula) y las de arriba (supparus). En el último caso, puede pensarse que la falsa etimología que relaciona supparus con supra- ha condicionado la creación de este segundo género de prendas que se ponen 'por encima' para justificar, precisamente, esta etimología. Sin embargo, Varrón vuelve a reiterar que sigue hablando de prendas que 'se ponen por encima' para incluir, junto a supparus, la palla (foris ac palam) y el indusium (intus). El pasaje puede resumirse con el esquema siguiente:

CUADro III. Varr. Ling. 131 (1)

indutui $\left\{\begin{array}{l}\text { capitium } \\ \text {-subtus (primum genus) subucula } \\ \text {-supra (alterum genus) supparus, palla, indusium }\end{array}\right.$

Así las cosas, una lectura del pasaje a partir de la disposición propia del mismo revela los hechos siguientes:

- Por muy incoherentes que resulten las etimologías dadas en el texto, si realizamos una lectura a partir de la propia ordenación del pasaje, observamos que la posición del indusium entre las prendas que se ponen supra es consecuente con la que veíamos en Varr. in Non. p. 870 L. (2):

Cuadro IV

\begin{tabular}{|l|l|l|}
\hline & \multicolumn{1}{|c|}{ Varr. in Non. p. 870 L. (2) } & \multicolumn{1}{|c|}{ Varr. Ling. V 131 (1) } \\
\hline $\begin{array}{l}\text { Prenda puesta por } \\
\text { encima }\end{array}$ & indusium & indusium (supra) \\
\hline $\begin{array}{l}\text { Prenda puesta por } \\
\text { debajo }\end{array}$ & subucula & subucula (subtus) \\
\hline
\end{tabular}

- Hay otro hecho, por lo demás, relacionado con lo que ya hemos visto en la diferencia entre inducula y subucula (1.2). Debe repararse en que lo 'interior' (intus), la etimología concreta dada por Varrón a indusium en el pasaje (1), no es necesariamente lo 'inferior' (subtus, inferus), aunque una y otra noción tiendan a ser coincidentes, en espacial dentro de los contextos indumentarios ${ }^{36}$. La diferencia entre una y otra noción puede verse claramente en el hecho de que sean nociones 'alternas' (o, en términos de la semántica tradicional, 'antónimas') distintas: así, intus se opone a extra, 'lo exterior' (cf.

${ }^{36}$ Éste es el caso de la interula (cf. Potthoff, op. cit., pp. 127-128), túnica interior, como la subucula, pero en este caso la denominación está formada a partir del adjetivo interulus (cf. Tert. pall. V 3: si quid interulae subter est, uacat zonae tormentum). Así pues, lo interior ha coincidido con lo inferior, como puede colegirse, igualmente, del testimonio ya citado de Nonio (4) intra plurimas uestes. 
Caes. Ciu. III 69, 4), mientras que subtus se opone a supra 'lo superior' (cf. Varr. $R$. III 10,4). Todavía en latín clásico es pertinente, por un lado, la distinción entre 'vestidos interiores', es decir, los más cercanos al cuerpo (uestes induendae) y 'vestidos exteriores', o aquellos que lo rodean (uestes amiciendae) y, por otro, dentro de las uestes induendae, entre la 'túnica superior' y la 'túnica inferior':

\section{CUADro V}

$\begin{array}{ll}\text { VESTES AMICIENDAE (EXTRA) } & \text { toga, pallium } \\ \text { VESTES INDVENDAE (INTVS) } & \left\{\begin{array}{l}\text { indusium (TVNICA SVPERIOR) } \\ \text { subucula } \text { (TVNICA INFERIOR) }\end{array}\right.\end{array}$

- Por otra parte ${ }^{37}$, el supparum, que si bien es una prenda del tipo indutui, no resulta tan clara, como ha hemos visto en 1.3 , respecto a su carácter de prenda que queda por encima (similar al indusium) o por debajo (similar a la subucula), nos hace reflexionar acerca de la arbitrariedad del propio signo lingüístico, agravada todavía más cuando se trata de las prendas con nombre extranjero. Es posible que el supparus, término griego venido al latín a través de los oscos en unión a algún tipo de prenda o, al menos, objeto textil, haya servido para realizar cualquier tipo de suposición etimológica supra-/sub-) por absurda que ésta fuera, y que los lexicógrafos tardíos acabaran confundiéndolo con la subucula por mera afinidad formal. Sin embargo, observando los testimonios literarios citados por Nonio en su definición del supparus (8), uno de ellos del propio Varrón, parece que se trata más bien de una túnica superior:

${ }^{37}$ En lo que a la palla respecta, se trata de una prenda de adscripción dudosa con respecto a su carácter amictui o indutui, pues, aunque en los testimonios que de ella conservamos aparece combinada con induo (Plaut. Men. 511-514, Rhet. Her. IV 60, Ov. Met. IV 483 y XIV 262-263 y Fast. 107) parece que, en principio, la palla debe de ser una prenda del tipo de las togas o los palios. En este sentido, tenemos un pasaje de Horacio donde se combina precisamente con el verbo circumdo, expresando, pues, la idea de movimiento 'en derredor':

Hor. S. I 2, 99: ad talos stola demissa et circumdata palla.

Marquardt (op. cit., p. 559; cf. también Ch. Daremberg y Edm. Saglio, Dictionnaire des Antiquités Grecques et Romaines, París, 1919, s. u. pallium, p. 292) hace un interesante comentario con respecto al carácter de la misma, tal y como podemos colegir a partir de los testimonios literarios, pues en unos casos aparece como indumentum ('vestido'), y en otros como amictum 'manto'. Por su parte, E. M. (s. u.) nos comentan una interesante pecularidad de la palla, el hecho de que se pliegue antes de ponerse, lo cual afecta igualmente a la manera en que ésta se coloca. No obstante, parece preferible pensar que la palla, más que un vestido mixto, presentara dos modalidades distintas, y que la palla como indumentum fuera privativa de dioses, héroes, personajes heroicos, adivinos o citaristas (García Jurado, op. cit., pp. 43-44). 
(12) $(=8)$ Non. p. 866 L.

a. Nouius Paedio (70)

supparum purum, Veliense interim-escam meram!

b. Afranius Epistula (122)

puella non sum, supparo si induta sum?

c. Varro Eumenidibus (121)

hic indutus supparum

coronam ex auro et gemmis fulgentem gerit.

En principio, porque esta prenda caracteriza a las personas que la llevan, tal y como colegimos del supparus de Velia procedente del pasaje del Novio (12 a), y si caracteriza a una persona es probable que la prenda se vea. Pero el principal argumento a favor nos lo da Varrón, esta vez como autor de las Sátiras Menipeas, pues coincide con la caracterización que hace del supparus como gramático al ver en él una prenda superior. Veamos el fragmento en su versión completa:

(13) (=12c) Varr. Men. 121: aurorat ostrinum hic indutus supparum / coronam ex auro et gemmis fulgentem gerit, / luce locum afficiens.

No hay duda alguna de que se trata de una prenda superior y muy visible, como el lujoso color ostrinum y los destellos (aurorat) dan a entender, de forma que la persona que va vestida con esta túnica 'jette les feux de l'aurore', según la traducción de Jean-Pierre Cèbe ${ }^{38}$.

\section{Conclusión}

Entendemos que el pasaje de Varrón Ling. V 131-133 (1) presenta una serie de aspectos esenciales para el estudio de las túnicas romanas:

- Las nociones 'intromisión' y 'en derredor' presentan en el lenguaje indumentario del latín arcaico y clásico una importancia que, en nuestra opinión, ha sido infravalorada por los estudiosos de los asuntos concernientes al vestido, quienes habitualmente las identifican, sin más, con las nociones 'por debajo' y 'por encima'. El estudio de las túnicas pone de relieve la pertinente diferencia entre unas nociones y otras, como puede verse representado en el cuadro siguiente, donde las diferentes túnicas se caracterizan por un rasgo común de

${ }^{38}$ Cf. Cèbe en su edición bilingüe comentada de las Sátiras Menipeas, Roma 1977, p. 533, quien comenta también: «Sur une robe talaire à manches longues, ils passaient un pallium pourpre - le supparum de notre texte - qui ressemblait à la chape des archiprêtes de la déesse syrienne» (p. 636), aunque parece que el propio carácter superior de la prenda le lleva a identificarla con una prenda amictui, en este caso un pallium. 
'intromisión' (frente a las uestes amiciendae) y un rasgo diferenciador en cuanto a su posición 'por encima' o 'por debajo':

\begin{tabular}{|l|c|c|}
\hline & intromisión indutui & por debajo subter \\
\hline indusium (inducula) & + & - \\
\hline supparus & + & - \\
\hline subucula & + & + \\
\hline
\end{tabular}

- La disposición del pasaje, que establece dentro de las prendas indutui un primer tipo subter y un segundo tipo supra, permite observar una caracterización del indusium y la subucula coincidente con el otro pasaje varroniano clave para el estudio de este tipo de prenda (2).

- La caracterización del supparus como un vestido indutui y superior coincide, a pesar de las divergencias con los lexicógrafos tardíos, con la caracterización que puede deducirse de los testimonios literarios, y en especial la de un testimonio varroniano de las Sátiras Menipeas (13).

Estos hechos presentan, en nuestra opinión, al testimonio varroniano como reflejo de un sistema indumentario concreto.

Francisco García Jurado 